\title{
ASSESSING THE PREVALENCE OF PARANASAL SINUSES ANATOMICAL VARIANTS IN PATIENTS WITH SINUSITIS USING CONE BEAM COMPUTER TOMOGRAPHY
}

\section{RALUCA ANCUTA ROMAN ${ }^{1}$, MIHAELA HEDEŞIU ${ }^{1}$, MIRELA GERSAK ${ }^{2}$, FLOAREA FIDAN ${ }^{1}$, GRIGORE BĂCIUT⿱ ${ }^{1}$, *MIHAELA BĂCIUȚ ${ }^{1}$}

\author{
${ }^{1}$ Department of Cranio-Maxillofacial Surgery, Faculty of Dentistry, Iuliu \\ Hatieganu University of Medicine and Pharmacy, Cluj-Napoca, Romania \\ ${ }^{2}$ Radiology Department, Clinical and Emergency County Hospital Cluj-Napoca, \\ Romania \\ * this author has the same contribution as the first author
}

\section{Abstract}

Background and aims. To asses, by using the Cone Beam CT (CBCT) reformatted images, the presence of anatomical variants of the sinonasal cavities and to determine the correlation of these variations with the onset of maxillary sinus inflammations.

Method. The study is a retrospective one and consists of the investigation of 130 patients with CBCT imaging, patients that were referred to the Maxillo-Facial Clinic, Radiology Department of the Iuliu Hatieganu University of Medicine and Pharmacy in Cluj-Napoca, for clinical symptoms of sinusitis within a period of 24 months. The images were analyzed for the presence of different anatomical variations and sinus inflammation. The CBCT images were obtained using a NewTom $3 G$ scanner and the data acquired were statistically analyzed using Chi-square test, Odds ratio data and confidence intervals, with a determined $p<0.05$ considered to be statistically significant.

Results. The anatomical variants were detected both in the inflammation and control group. From the spectrum of variations concha bullosa, deviation of uncinate process and asymmetrical ethmoid roof presented significant association with sinusitis. The deviated position of the uncinate process appeared in more than $50 \%$ of patients in the positive group $[O R=2.55]$ compared with a third of the control group. Concha bullosa was observed in 31\% cases, 23\% in the control group and 34\% in the positive group $[O R=1.73]$. Haller cells showed a small difference between groups [OR=1.14] whereas the ethmoid roof asymmetry was evidently more prevalent in the inflammation group.

Conclusion. The anatomical variants of the paranasal sinuses are not incidental, being found in a large number of patients and may be a predisposing factor in the onset and recurrence of sinuses inflammation. The CBCT technique, due to the finest multiplanar reconstruction, permits a very good pre-therapeutic assessment of these predisposing conditions.

Keywords: CBCT, maxillary sinuses, anatomical variants

Manuscript received: 15.10 .2015

Accepted: 24.11.2015

Address for correspondence: mhedesiu@gmail.com

\section{Background and aims}

The imaging investigation of the anatomical variation of the paranasal sinuses is important in assessing the predisposing factors for inflammatory changes of the 
paranasal sinuses. These changes of the sinuses are a common problem encountered in clinical practice.

Especially for the functional endoscopic surgery and its large use in treating chronic sinusopathy refractory to medication, optimal evaluation of this region is of great importance for the safety and success of the procedure. Nouarei et al. in their study on 278 patients in 2009 concluded that the anatomic variants of the bone structures were not increasing the risk to develop mucosal disease of the sinus but they cannot exclude the potential impact on the safety of the surgery. Therefore these anatomic variations need to be assessed in the preoperative evaluation [1]. Computer Tomography is considered the method of choice due to its best approach of the bone structures. For assessing correctly the paranasal structures, with a special focus on the osteo-meatal unit, the coronal approach is of utter importance. The use of Cone Beam CT technique, with its high-quality bone definition, is considered an advancement to $\mathrm{CT}$ and can provide, by using very thin and multi-planar cross-sectioning, a very good approach on scanning the maxillary structures, especially regarding the paranasal cavities and the implications of their position, shape and variations in the inflammatory pathology [1-7].

The paranasal sinuses development, through the process of the nasal mucosa invagination in the surrounding facial bones, explains the high number of anatomical variations, whether we talk about supplementary cavities or about different shape, different position or insertion of some fine structures $[2,3]$.

The most encountered variations are the concha bullosa, Haller cell presence and the modifications of the uncinate process morphology and positions, according to the Al-Abri et al. study performed on 453 patients and reported in 2014, Perez-Pinas study in 2000 and Smith's study in $2010[6,7,8]$. Concha bullosa or the middle turbinate pneumatization is a common finding, and when large, it is accompanied by a deviation of the nasal septum, due to developmental asymmetry [9-18]. Studies as of Pérez-Piñas et al. and Smith et al. on the relationship with inflammation showed little clinical importance of this variation, mainly existing without symptoms, but the pathology of this air cavity can be present through infection or obstruction. Similar appearances may be seen also in the inferior or superior turbinate [6,7].

The Haller cell is an asymptomatic maxilloethmoidal cell, extramural one that extends into the orbital wall inferior and medial and in some situations may narrow the osteo-meatal complex predisposing to the onset of sinusitis through obstruction of the maxillary sinus drainage [7-10].

The uncinate process was largely studied, being a major part of the osteo-meatal unit that allows air flow and also mucus drainage. Morphological variations of this hook-like process, that forms the anterior part of the hiatus semilunaris, can be a factor of narrowing the unit thus blocking the drainage and consequently producing inflammation [12-14,16-19]. Other anatomical variations are the sphenoethmoidal Onodi cell, a rare posterior aerated cell, closely related to the optic nerve and the internal carotid artery, thus constituting a risk factor in surgical approaches, a large Agger nasi cell, which is the most anterior ethmoidal cell, or an asymmetric ethmoidal roof.

Several previous studies of these anatomical variations used computer tomography to detect the correlation with sinus inflammation. Few studies used CBCT as a low irradiating imaging tool, such as the one of Riello et al., Mathew et al., with different results in assessing these aspects, due to different series of cases or approach $[2,7,14,19]$.

The aim of this study was to assess, by using the Cone Beam CT (CBCT) reformatted images and multiplanar approach the presence of anatomical variants of the sinonasal cavities, and to determine the correlation of these variation with the onset and recurrence of maxillary sinus inflammations.

\section{Material and methods}

A retrospective cohort study was conducted consisting of 130 patients investigated clinically and with Cone Beam CT imaging. All patients were referred to the Maxillofacial Radiology Department, in a 24 months period between 2011-2012, for clinical suspicion of sinusitis. A control group of 27 patients with CBCT examination for implant planning, without clinical symptoms and with a normal aspect of paranasal sinuses was also included in the study.

The CBCT images were obtained using a NewTom 3G scanner (Quantitative Radiology, Verona, Italy, pixel size: $0.180 \mathrm{~mm}$ ). Two radiologists, specialized in maxillofacial radiology, examined the images using the default NNT viewer. The axial, coronal and sagittal planes were used to evaluate the following anatomical structures and their variations: (1) the middle turbinate morphology, for example inverted middle turbinate which represents paradoxical turbinate directed towards medial of maxillary sinus [figure 1a], or the presence of concha bullosa which represents the aerated middle turbinate [figure 1b], (2) the aspect, shape, dimension, position and insertion of the uncinate process [figure 3, figure 4, figure 5] (3) nasal septum deviation [figure $1 \mathrm{a}, \mathrm{b}$ ], (5) presence of Haller cells, an infraorbital air cell, called also the maxillo-ethmoidal cavity [fig 2], (6) presence of Onodi cells [figure 7], (7) the aspect and dimension of the anterior Agger nasi cell, (8) the presence of an asymmetry of the ethmoid roof [figure 6].

The thickness of the maxillary mucosa was measured on CBCT sections and inflammation was considered to be present at a thickness above $2 \mathrm{~mm}$. Different aspects of the mucosa were also assessed, as polipoid thickening, retention cyst or completely opaque sinus due to large fluid retention. 

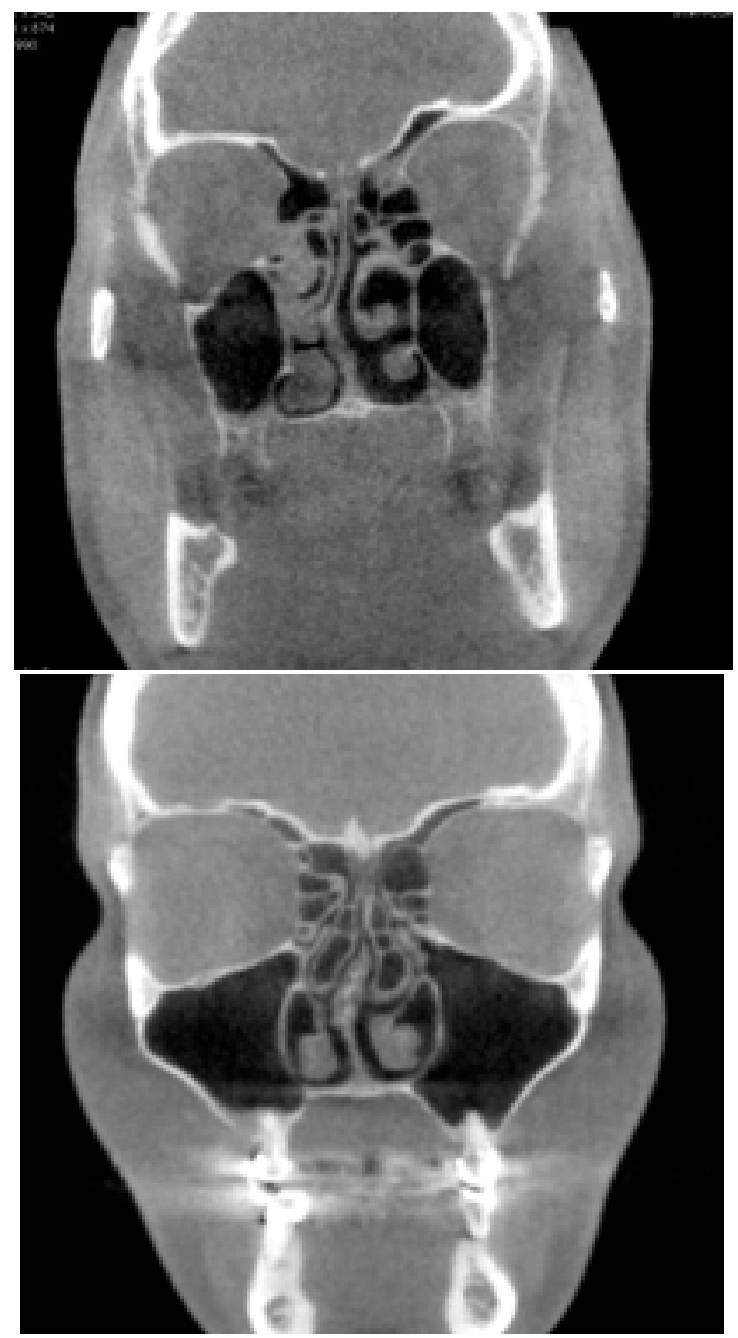

Figure 1 a,b. Midlle turbinate variations a. Right inverted middle turbinate with a right nasal septum deviation. b. Bilateral pneumatized middle turbinate associated right nasal septum deviation.

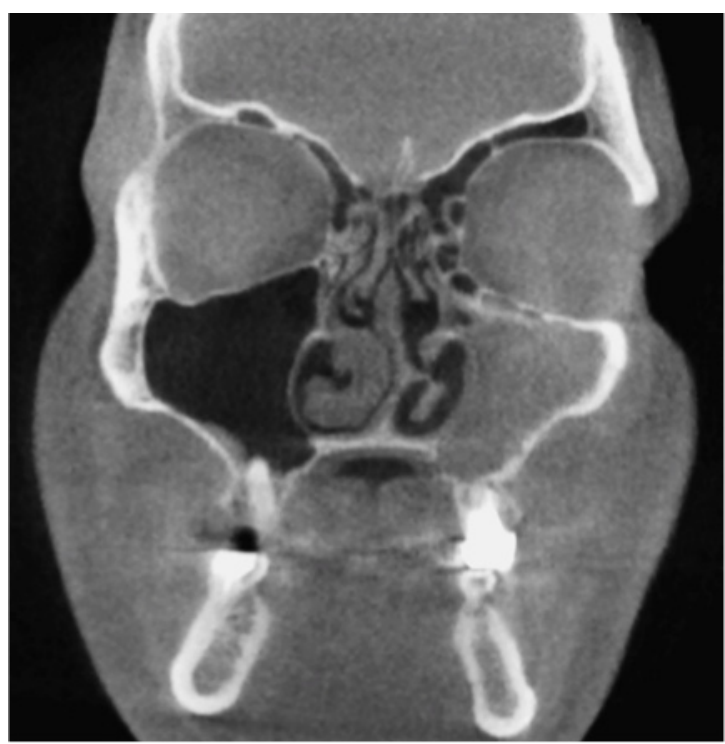

Figure 2. CBCT coronal section. Left visibly occupied Haller cell and right inverted middle turbinate. Left sinus inflammation.

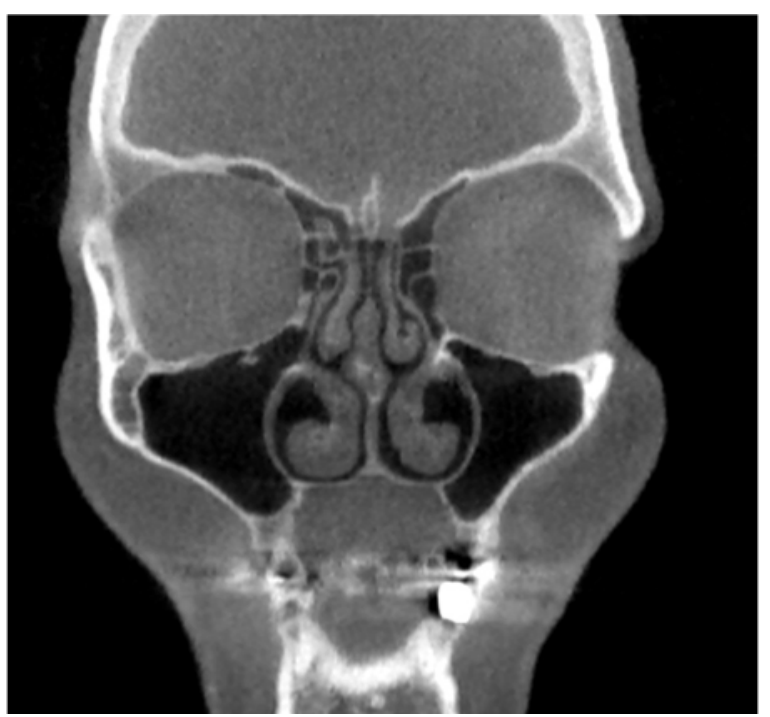

Figure 3. CBCT coronal section. Pneumatized uncinate process (UP).

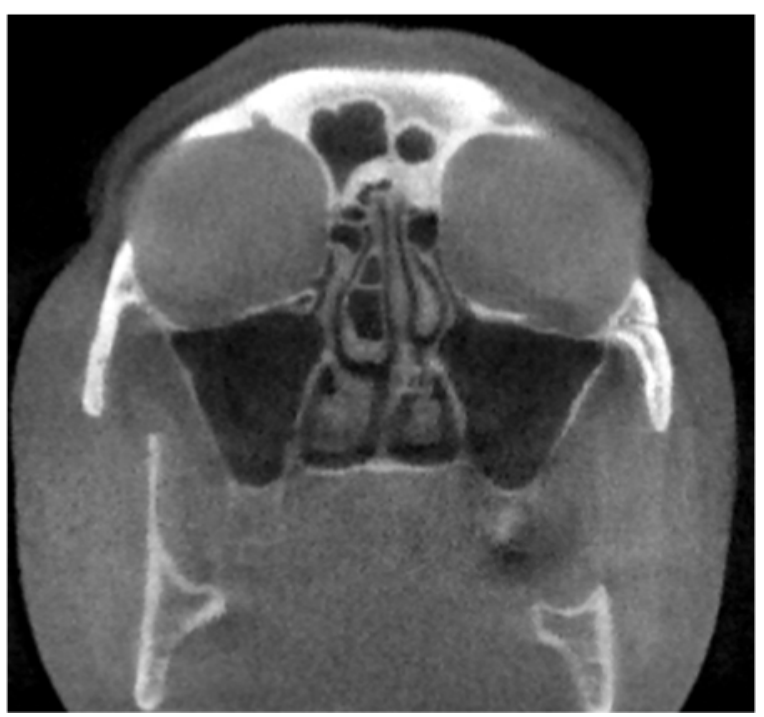

Figure 4. CBCT coronal section. Cribriform plate attachment of the uncinate process in a case with ipsilateral concha bullosa.

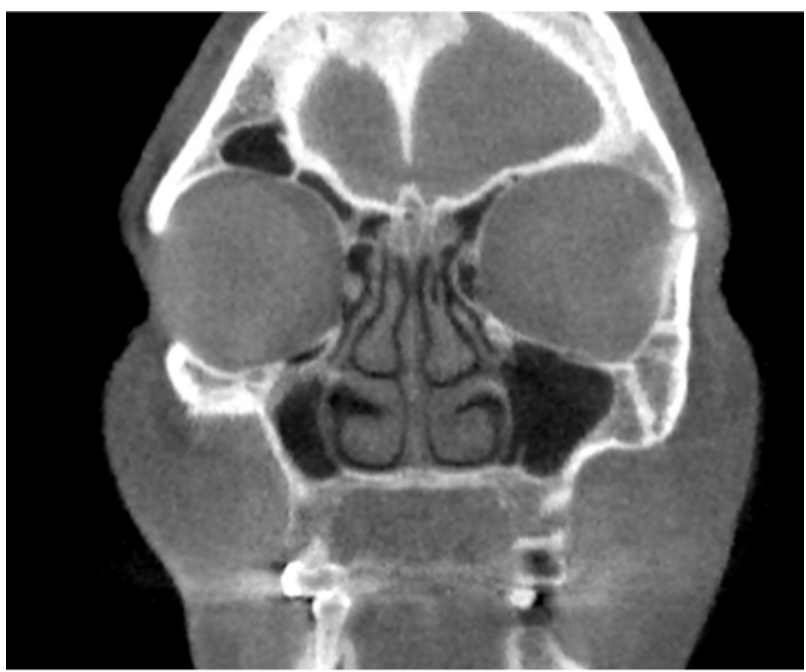

Figure 5. CBCT coronal section, superior insertion of the uncinate process. 


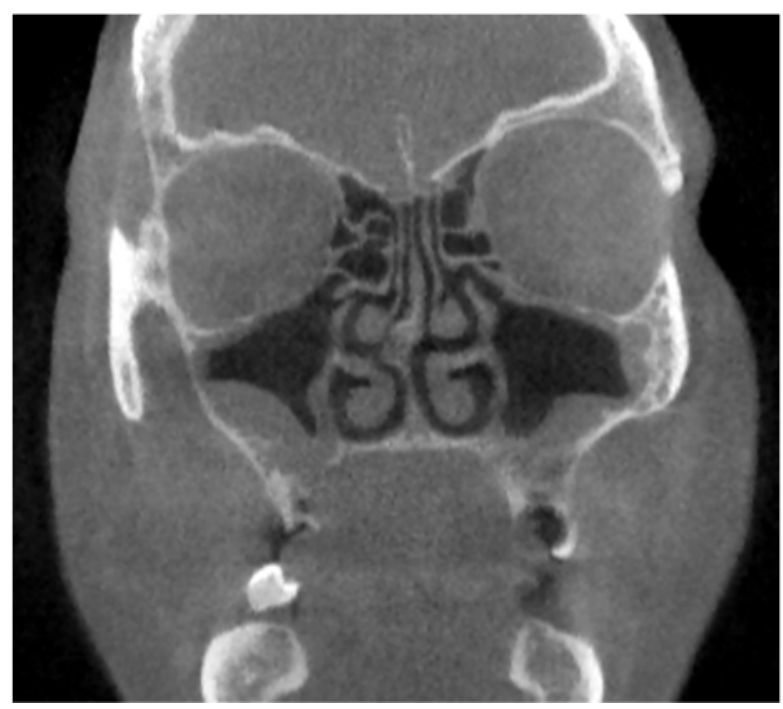

Figure 6. CBCT coronal view. Ethmoidal roof asymmetry with bilateral maxillary sinus inflammation.

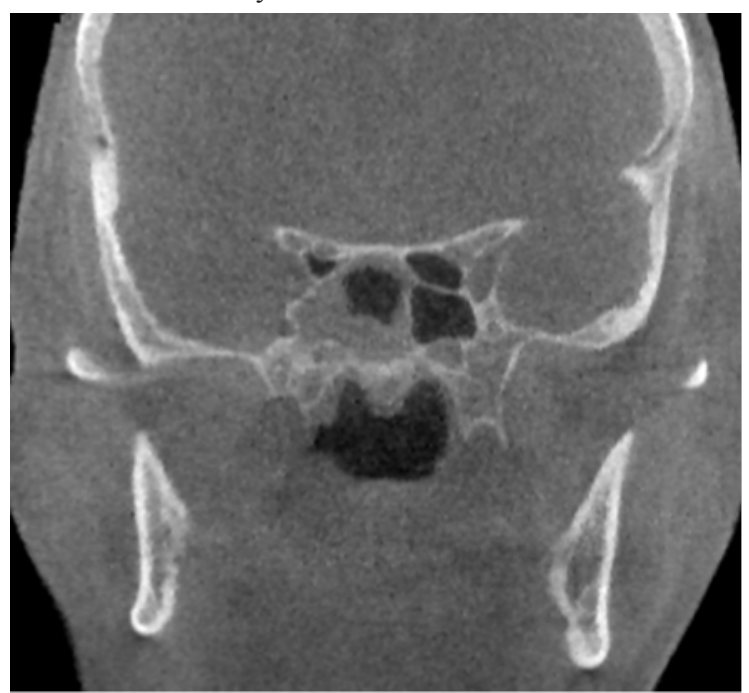

Figure 7. CBCT coronal section. The presence of Onodi cells in conjunction with right sphenoid sinus.

The prevalence of the maxillary sinus anatomic variants and their correlation with the thickness of the mucosa were statistically assessed, using the Fischer exact test, Odds ratio data and confidence intervals, with a determined $p<0.05$ considered to be statistically significant.

We compared our results with studies published in the literature that aimed to detect the same anatomical variations using $\mathrm{CT}$ or $\mathrm{CBCT}$ examination. These studies investigated the presence of all these variations or only of some of them and their implication in the sino-nasal inflammatory pathology.

\section{Results}

A number of 130 CBCT images, 61 females and 69 males, with the mean $\pm \mathrm{SD}$ age of $37.63 \pm 13.5$ years were assessed. The anatomical variants were noted in both groups. The incidence of the anatomic variants among each gender group were sensibly close, presenting no statistical significance.

The most common anatomic variant in our study as the asymmetry of the ethmoid roof ( $62 \%$ of all subjects) followed by the middle turbinate variants $(58 \%)$, the middle pneumatized concha (35.3\%) and Haller cell (25\%). Table I presents the overall results of the present study comparatively with previous studies.

The most common variation encountered was the asymmetry of the ethmoid roof in $62 \%$ of all the cases, followed by the middle turbinate modifications, present in $58 \%$ of all subjects.

These changes regarded the morphology or the dimension. From all the anatomic variants of the middle turbinate the most prevalent ones were the concha bullosa and the inverted turbinate aspect. Inverted middle turbinate was detected in our study in $8 \%$ of the cases while concha bullosa, was present in $35.3 \%$ of the cases, with a significant difference between the positive and control group (42\% to $27 \%$, Fischer exact test $\mathrm{p}=0.0369$ ).

The Haller cell was identified in $27 \%$ of cases in the positive group and in $19 \%$ in the control group, with a Fischer exact test results $p$ of 0.01 .

Anatomical variation in the aspects of the osteomeatal unit were present in $35 \%$ of the positive group along with the presence of mucosal thickening in $80 \%$ of these cases, with a Fischer exact test $\mathrm{p}=0.01$. In the control group the osteo-meatal unit anatomic aspects were present in $15 \%$ of the cases, OR of 2.5568. The variations in the attachment of the uncinate process were investigated, and the superior insertion with a cribriform attachment appeared in $42 \%$ cases in the control group and in $46 \%$ in the positive one, with an OR of 0.94 , and a Relative Risk: $1.7287,95 \%$ CI [1.2906-0.0244].

Other modifications encountered were pneumatization of the uncinate process, or deviated position of the uncinate process from normal, encountered in $30 \%$ of the patients in the control group and in $53 \%$ in the positive group, with OR of 2.55. and a value $\mathrm{p}=0.0065$. Detailed data results are presented in table II regarding the main anatomical findings.

The ethmoid roof asymmetry was present in 80 patients, $81 \%$ of them in the positive group whereas $24 \%$ in the control group, $\mathrm{p}=0.0001$.

Onodi cell was visualized in $4 \%$ of the cases in the control group and $6 \%$ in the positive one, sphenoid sinus inflammation was detected in $10 \%$ of the cases in the positive group.

The anterior ethmoid Agger nasi cells were seen in $93.2 \%$ of the cases being the most common anatomic variation. 
Table I. Anatomical variations of the paranasal sinuses and their prevalence in previous studies.

\begin{tabular}{|c|c|c|c|c|c|c|c|c|}
\hline & $\begin{array}{l}\text { Present } \\
\text { study }\end{array}$ & $\begin{array}{l}\text { Adeel } \\
\text { et al. } \\
2013 \\
{[10]}\end{array}$ & $\begin{array}{l}\text { Fadda } \\
\text { et al. } \\
2012 \\
{[5]}\end{array}$ & $\begin{array}{l}\text { Perez et } \\
\text { al. } \\
2000 \text { [6] }\end{array}$ & $\begin{array}{l}\text { Dutra } \\
\text { et al. } \\
2002 \\
{[12]}\end{array}$ & $\begin{array}{l}\text { Mamatha } \\
\text { et al. } \\
2010 \\
{[13]}\end{array}$ & $\begin{array}{l}\text { Mazza } \\
\text { et al. } \\
2007 \\
{[11]}\end{array}$ & $\begin{array}{l}\text { Nouraei } \\
\text { et al. } \\
2009 \\
{[1]}\end{array}$ \\
\hline Concha bullosa & $35.3 \%$ & $18.2 \%$ & $2,8 \%$ & $24,5 \%$ & $4.2 \%$ & $15 \%$ & $29 \%$ & $35 \%$ \\
\hline $\begin{array}{l}\text { Paradoxical } \\
\text { middle turbinate }\end{array}$ & $8 \%$ & $14.3 \%$ & $6.4 \%$ & $10 \%$ & - & - & $11 \%$ & - \\
\hline $\begin{array}{l}\text { UP } \\
\text { pneumatisation }\end{array}$ & $5 \%$ & $5.2 \%$ & $2.8 \%$ & none & - & - & $5 \%$ & - \\
\hline Haller cell & $25 \%$ & $9,1 \%$ & $22.8 \%$ & $45 \%$ & $1.4 \%$ & $17.5 \%$ & $5 \%$ & $12 \%$ \\
\hline Onodi Cells & $8 \%$ & $7.8 \%$ & $8.5 \%$ & $10.9 \%$ & - & - & $9 \%$ & - \\
\hline $\begin{array}{l}\text { Asymmetry of } \\
\text { ethmoidal roof }\end{array}$ & $62 \%$ & - & $25.7 \%$ & - & - & - & - & $11 \%$ \\
\hline No. of cases & 130 & 77 & 140 & 110 & 71 & 40 & 100 & 278 \\
\hline
\end{tabular}

Table II. Results for the significant variation encountered.

\begin{tabular}{llll}
\hline Anatomical variation & $\begin{array}{l}\text { control } \\
\text { group }\end{array}$ & $\begin{array}{l}\text { positive } \\
\text { group }\end{array}$ & $\begin{array}{c}\text { p } \\
\text { (Fischer exact test) }\end{array}$ \\
\hline Haller cell & $19 \%$ & $27 \%$ & 0.01 \\
\hline Concha bullosa & $27 \%$ & $42 \%$ & 0.0369 \\
\hline UP variation & $15 \%$ & $35 \%$ & 0.01 \\
\hline deviated UP & $30 \%$ & $53 \%$ & 0.0065 \\
\hline ethmoid roof asymmetry & $24 \%$ & $81 \%$ & 0.0001 \\
\hline
\end{tabular}

\section{Discussion}

The presence of the anatomical variations of the maxillary sinus are not uncommon and are more frequently detected, according to previous studies in patients with sinusitis than in the healthy population. Therefore, the identification of the anatomical variation could be more important in the evolution of the recurrent sinuses inflammation, due to their implication in the drainage of the fluid from the sinuses cavities.

Computer Tomography and more recently CBCT offers valuable information regarding the anatomy of the maxillofacial region with a lower dose compared to CT.

The present study highlighted the presence of the paranasal sinuses anatomical variants on CBCT as an low level radiation alternative to the multi-slice CT used as the imaging tool in previous studies in the literature. The samples of cases are closely similar in number with the ones presented in the literature, the difference being in the number of anatomical variations assessed. Our study highlights all variations of the paranasal sinuses and their detection using CBCT in helping the clinician or the surgeon in their work.

In our study the incidence of concha bullosa was significantly related with the presence of mucosal thickening, results similar with the ones reported by Nouraei et al., Riello et al. and Perez et al. [1,6,19], being present in one third of the patients. The incidence under $5 \%$, presented by Fadda et al. and Dutra et al. $[5,12]$ are in opposition with our results, facts possibly explained by the type of examination, CT versus CBCT in our study or the sections used. Unlike previous studies which used the reconstructed coronal plane from the $\mathrm{CT}$, CBCT offered the possibility to assess this anatomical region on different numbers of inclinations, in order to exquisitely appreciate the area, and possible modifications from the well- known normal aspect. Our study differs also from the Smith et al. one with regard to concha bullosa presence, their study stating no apparent statistically significant relationship between the presence of this modification and maxillary sinusitis. Our cases of concha bullosa were mostly related with nasal septum deviation, fact that sustains the implication in the onset of inflammation.

In concordance with main studies in the literature, with an OR of 1.1 and a $\mathrm{p}$ value of 0.01 , the results on the Haller cells presence demonstrate the implication of this cell in the onset of maxillary sinus inflammation, with results significantly different between groups [5]. There are also on this matter studies that contradict the results and sustain that there is no significant relationship with chronic rhino-sinusitis, as does the study of Mathew et al. [15].

The most significant relationship between a variation in anatomy of this region and the presence of 
chronic sinuses inflammation proved to be the one with the asymmetry of the ethmoid roof, which was present in more than $80 \%$ of the cases with sinusitis, with a p of 0.0001 . The study reported by Fadda et al. investigated this relationship, and their results are not in line with ours, depicting this variation in only $25 \%$.

The incidence of paradoxical middle turbinate in this study was $8 \%$ which is in agreement with the studies performed by Perez et al., Fadda et al. and Maza et al. $(10 \%)$, and closely with the result of the Adeel et al. study, which was $14 \%[5,6,10,11]$. Only few of the cases with this variation presented mucosal inflammation, some of them on the contralateral site $(25 \%)$ data in agreement with previous studies, not proving to have any implication in the presence of mucosal inflammation.

One of the most important parts in the path of mucus drainage consists in the osteo-meatal unit, where the uncinate process, owing to an abnormal shape or position, can become a very important favoring factor in the appearance of sinusitis. These variations, in position or in shape were depicted in a third of our positive group $(\mathrm{p}<$ 0.05 ), in opposition with the control group, and consisted mainly in variation of the position, in half of the group, followed by deviation in the insertion. All these variations proved significant statistical relationship with sinusitis, in agreement with the Fadda et al. and Azila et al. studies $[5,16]$.

Riello and Boasquevisque conclude in their study on $\mathrm{CT}$ detection of the osteo-meatal complex and nose anatomical variations in 50 patients that these situations are common, most frequently regarding the middle turbinate and the nose septum [19].

The presence of the Onodi cell, in few cases and similar percentages between groups was not significant in the onset of inflammation but due to its close anatomical site to nerve and arterial path should be assessed before any surgery involving the area of sphenoid sinus.

The Agger nasi cell was present in almost all patients investigated, more than $90 \%$, their appearance not proving any relationship with mucosal inflammation, even though different studies showed variable results, both in the presence (from $2.6 \%$ till 97\%), and in the dimension, and sustained its implication, at least what ethmoid sinusitis is concerned. In our study we cannot conclude to the relationship between mucosal reaction and a possible cause brought by an Agger nasi modified cell.

There are limitations of the present study, mainly due to the fact that the population does not accurately represent the general population, and because all patients were referred to CBCT for suspected sinusitis. This way the forming of the control group was difficult and with a rather small number of subjects. These limitations are difficult to overcome because a study with a truly randomized population would require subjects to undergo unnecessary exposure to radiation and thus contradict with the ALARA principle,"as low as reasonable achievable".

\section{Conclusions}

- Some anatomic variants of the paranasal sinuses may constitute a predisposing factor in the appearance and recurrence of chronic sinuses inflammation.

- The concha bullosa, the deviation position of the uncinate process and the asymmetrical ethmoid roof proved significantly association with sinusitis, and needs to be detected in management of a patient with chronic sinusitis for a good prognosis.

- The CBCT examination, using lower radiation doses than multislice-CT, due to the finest multi-plane reconstruction, allows for the best pre-therapeutic assessment of these anatomic/predisposing conditions.

\section{References}

1. Nouraei SA, Elisay AR, Dimarco A, Abdi R, Majidi H, Madani $\mathrm{SA}$, et al. Variations in paranasal sinus anatomy: implications for the pathophysiology of chronic rhinosinusitis and safety of endoscopic sinus surgery. J Otolaryngol Head Neck Surg. 2009;38(1):32-37.

2. Miracle A, Mukherji SK. Conebeam CT of the head and neck, part 2: clinical applications. Am J Neuroradiol. 2009;30:12851292.

3. Limeme M, Benzina M, Majerbi M, Kheireddine N, Zaghouani $\mathrm{H}$ et al. Anatomical variants in the sino-nasal region: a pictorial review. EPOS. ECR 2015. C-2406. doi:10.1594/ecr2015/C-2406 4. Alkire BC, Bhattacharyya N. An assessment of sinonasal anatomic variants potentially associated with recurrent acute rhinosinusitis. Laryngoscope. 2010; 120: 631-634.

5. Fadda GL, Rosso S, Aversa S, Petrelli A, Ondolo C, Succo G. Multiparametric statistical correlations between paranasal sinus anatomic variations and chronic rhinosinusitis. Acta Otorhinolaryngol Ital. 2012; 32(4): 244-251.

6. Pérez-Piñas, Sabaté J, Carmona A, Catalina-Herrera CJ, Jiménez-Castellanos J. Anatomical variations in the human paranasal sinus region studied by CT. J Anat. 2000;197:221-227. 7. Smith KD1, Edwards PC, Saini TS, Norton NS. The prevalence of concha bullosa and nasal septal deviation and their relationship to maxillary sinusitis by volumetric tomography. Int J Dent. 2010;. doi: 10.1155/2010/404982

8. Al-Abri R, Bhargava D, Al-Bassam W, Al-Badaai Y, Sawhney S. Clinically significant anatomical variants of the paranasal sinuses. Oman Med J. 2014;29(2):110-113.

9. Stallman J, Lobo JN, Som P. The incidence of concha bullosa and its relationship to nasal septal deviation and paranasal sinus disease. Am J Neuroradiol. 2004;25:1613-1618.

10. Adeel M, Rajput MS, Akhter S, Ikram M, Arain A, Khattak YJ. Anatomical variations of nose and para-nasal sinuses; CT scan review. J Pak Med Assoc. 2013;63(3):317-319.

11. Mazza D, Bontempi E, Guerrisi A, Del Monte S, Cipolla G, Perrone A, et al. Paranasal sinuses anatomic variants: 64-slice CT evaluation. Minerva Stomatol. 2007;56:311-318.

12. Dutra LD, Marchiori E. Helical computed tomography of the paranasal sinuses in children: evaluation of sinus inflammatoy diseases. Radiologia Brasileira. 2002;35:161-169.

13. Mamatha H, Shamasundar NM, Bharathi MB, Prasanna LC. Variations of ostiomeatal complex and its applied anatomy: a CT 
scan study. Indian J Sci Technol. 2010;3:904-907.

14. Khojastepour L, Mirhadi S, Mesbahi S. Anatomical Variations of Ostiomeatal Complex in CBCT of Patients Seeking Rhinoplasty. J Dent (Shiraz). 2015;16(1):42-48.

15. Mathew R, Omami G, Hand A, Fellows D, Lurie A. Cone beam CT analysis of Haller cells: prevalence and clinical significance. Dentomaxillofac Radiol. 2013;42(9):20130055.

16. Azila A, Irfan M, Rohaizan Y, Shamim AK. The prevalence of anatomical variations in osteomeatal unit in patients with chronic rhinosinusitis. Med J Malaysia. 2011;66:191-194.

17. Miranda CMNR, MaranhaoCPM, Arraes FMNR, Padiha IG,
Farias LPG, Jatoba MSA et al . Anatomical variations of paranasal sinuses at multislice computed tomography: what to look for. Radiol Bras. 2011;44(4):256-262.

18. Biswas J, Patil C, Deshmukh P, Kharat R, Nahata V. Tomographic Evaluation of Structural Variations of Nasal Cavity in Various Nasal Pathologies. International Journal of Otolaryngology and Head \& Neck Surgery. 2013;2(4):129-134. doi: 10.4236/ijohns.2013.24028.

19. Riello AP, Boasquevisque EM. Anatomical Variants of the Ostiomeatal Complex: Tomographic Findings in 200 Patients. Radiol Bras.2008.41(3):149-154. 